\title{
Editorials
}

\section{Anesthesia physician resources - time to change the focus}

\author{
Neil Donen MD FRCPC, ${ }^{*}$ Jean-François Hardy MD FRCPC $\dagger$
}

I $\mathrm{N}$ the past five years, five different articles on anesthesia physician resource planning have appeared in the Canadian Journal of Anesthesia (CJA). ${ }^{1-5}$ Each has taken a different approach. The first, authored by Donen et al., looked at total numbers of practicing anesthesiologists and related the findings to physician population ratios. ${ }^{1}$ Yang et al. used two models to assess anesthesia physician resources in Ontario, demand-based and benchmarking. ${ }^{2}$ Byrick approached the problem using a 'per capita' expenditure model as a measure of clinical services provided. ${ }^{3}$ In the same issue Craig et al. combined the per capita model with the Canadian Medical Association database and annual numbers of Royal College of Physicians and Surgeons of Canada certificants to reach their conclusion. ${ }^{4}$ In the current issue Engen and colleagues take a totally different approach. Their demand-based assessment of perceived anesthesia physician resource requirements is the result of a highly successful survey (93\% response rate) of all health care institutions in Canada providing anesthesia services. ${ }^{5}$ Despite these different approaches the message in each case is the same. There are insufficient numbers of anesthesiologists to meet the current and predicted future demands.

This problem is not unique to Canada. Reports from other countries document the same concern. ${ }^{6-9}$ The trend extends to the various surgical specialties in Canada who are experiencing similar shortages. ${ }^{\mathrm{A}}$ Adding to the picture are the decreasing numbers of nurses, both in Canada and the United States, who are an integral part of the delivery of surgical and anes-

A Adams $O$. Canadian Specialty Society recommendations: Categorical summation. Canadian Medical Association. February 2, 2004 thesia services. ${ }^{10,11}$ A recent British Columbia Medical Association report highlighted the specific understaffing of nurses in operating rooms, postanesthesia recovery and critical care units. ${ }^{12}$

Other factors have added to the complexity and mix of future physician resource planning. Etzioni, looking at the aging population in the United States, has conservatively estimated a $13 \%$ increase in the current surgical workload by 2010 and $30 \%$ by $2020 .{ }^{13}$ In May 2003 , the World Medical Association issued a statement on ethical guidelines for international recruitment of physicians indicating that richer countries should not undermine the physician resources of poorer and third world countries by actively recruiting physicians to meet their own needs. ${ }^{14}$ Many of these poorer countries have begun to actively lobby governments of wealthier countries, such as Canada, to restrict their inward flow of international physicians. One other additional, and poorly documented, factor has been the increase in severity and complexity of anesthesia patients over the past 20 years. This perception (shared by several colleagues), coupled with the increase in evening and night workload, redistribution of surgical and anesthesia services by health authorities and a much more impersonal approach to medical staff by administrators has added to the stress and burnout experienced by many Canadian anesthesiologists today.

Given the worldwide shortage of anesthesiologists we believe it is time for us to move from documentation of shortages to finding solutions that maximize the provision and delivery of anesthesia services. The recent Ottawa Federal/Provincial Health Summit in September 2004 provides us the opportunity to move in this direction. ${ }^{15,16} \mathrm{We}$ see this as a series of challenges to the Canadian anesthesia community and organizations such as the Canadian Anesthesiologists'

From the Department of Continuing Medical Education and Professional Development, Royal Jubilee Hospital, Victoria, British Columbia; and the Département d'anesthésiologie, Centre hospitalier de l'université de Montréal, Hôpital Notre-Dame, Montréal, Québec, Canada.

Address correspondence to: Dr. Neil Donen, Department of Continuing Medical Education and Professional Development, Vancouver Island Health Authority, 103 Begbie Hall, Royal Jubilee Hospital, 1900 Fort Street, Victoria, British Columbia V8R 1J8, Canada. Phone 250-370-8425; Fax: 250-370-8556; E-mail: ndonen@shaw.ca 
Society (CAS), the Association of Anesthesiologists of Quebec and the Association of Canadian University Departments of Anesthesia.

\section{Challenge \#1}

Canadian anesthesia organizations should spearbead a national process to develop a standardized approach to anesthesia physician resource data identification and collection for the purposes of documenting anesthesia physician resource needs in Canada

Seeking active solutions does not imply abandoning documentation of need. All approaches used so far have contributed to our knowledge. However, we must now develop a consistent model of measurement. This measurement may include some, or all, of the approaches taken so far. We must agree upon what we should measure, what to document, the ease and cost of measurement, its reliability and frequency of measurements. It must include the key players such as government and health planners. What is obvious is that the approach needs to be anesthesia specific.

\section{Challenge \#2}

Canadian anesthesia organizations, in addition to lobbying to improve working conditions for anesthesiologists, must develop a comprehensive physician health program for anesthesiologists

We have already alluded to issues of stress and burnout amongst colleagues. An unhappy workforce is unproductive and non-sustainable. It is also possible that the increased workload and stress may negatively impact on other issues such as patient safety. In addition to seeking to improve the working environment there is a critical need to develop educational and support programs that will assist the anesthesiologist in managing the stresses of daily work. This includes programs such as conflict resolution skills in the operating room and personal life style management techniques. These programs need to be widely disseminated and supported.

\section{Challenge \#3}

Canadian anesthesia departments need to undertake and publish studies relating to the effectiveness of pre-, intra- and perioperative processes

All five of the articles published in the CJA called for an increase in the number of anesthesia residency positions. Despite the fact that a number of medical schools are expanding their undergraduate and postgraduate quotas, realistically we will only be allocated a small number of residency positions. These will not be enough to meet the expected and ongoing demand for anesthesia services. Thus we will need to turn to other options and approaches.
Small but significant gains in the number of cases serviced by anesthesiologists can be achieved by looking how effectively we deliver these services pre- and perioperatively. Increasingly, articles are appearing from other countries on health systems research. ${ }^{17,18}$ No doubt similar work is being undertaken at various Canadian institutions. However there is no documentation of the effectiveness, or ineffectiveness of the various strategies attempted. The Institute for Healthcare Improvement has been a dramatic driving force in the area of quality improvement. Focused projects by workgroups from many North American institutions have achieved very laudable results. ${ }^{19}$ There is a critical need for us to study our anesthesia and surgical processes as well as to document and share successes and failures. To be successful these studies need to be undertaken jointly with our surgical and nursing colleagues.

In Quebec specially trained anesthesia assistants (AA; members of the Ordre Professionnel des Inhalothérapeutes du Québec) have played an important supportive role for many years. This has included preparing equipment for cases and assisting the anesthesiologist during induction and emergence. In selected instances they can supervise the case alone (albeit the anesthesiologist must remain immediately available) following induction of anesthesia by the anesthesiologist. Of note is that in 2004 the CAS endorsed the role and scope of practice of AA and amended their Guidelines to Practice of Anesthesia to reflect this. ${ }^{20}$ Recently in Ontario, AA have been introduced to assist in the operating room. The proponents of AA indicate their value in improving efficiencies of service delivery. Given the need to justify such an approach it may be useful to undertake a cost-effectiveness analysis of using AA in the operating suite.

A more controversial area for review is that of indications for surgery and anesthesia. Given the limited number of personnel available for anesthesia services we need to have a close look at the value and effectiveness of performing various procedures on some patients. This is an area of practice that is closely aligned with standards of care and quality improvement. We must visit and evaluate this issue.

\section{Challenge \#4}

Canadian anesthesia organizations should take a leadership role in exploring the potential and roles of nonmedical personnel in providing anesthesia services

Finally there is the issue of alternate providers in the technical and monitoring areas of delivered care. Over the years physicians have turned over some of these responsibilities to other health care providers as 
well as giving them increasing responsibility to intervene when appropriate. Well-known examples are prehospital emergency medical technicians and critical care nurses. In the United States of America nurse anesthetists (NA) have been providing anesthesia services for almost 120 years. Both in the United States and in many parts of Europe they function either independently or interdependently with an anesthesiologist as part of a team providing the anesthesia services. The implementation of the European working time directive, which limits the numbers of hours worked per week, has had a significant effect on the ability of anesthesiologists to deliver adequate services. As a result a number of countries are seriously looking at introducing NA to resolve the physician resource problem. Currently in the United Kingdom a number of pilot projects using NA are underway. ${ }^{7,21}$ The anesthesiology organizations are working very closely with the authorities so as to ensure that training programs, standards of care and future delivery of anesthesia care are closely tied to their own organizations. In this regard they are seeking to avoid the same mistake made by the American Society of Anesthesiologists in the early part of the last century when they sought to undermine the efforts of NA. This resulted in the subsequent establishment of an independent NA credentialing body and organization.

In Canada there has also been a subtle shift in this direction. Pre-admission clinics are increasingly using specially trained nurses to undertake pre-operative assessments. Many non-teaching hospitals now rely on family practitioners to complete the preoperative history and physical examination. The anesthesiologist most commonly meets the patient for the first time literally minutes before the anesthetic. While well accepted by the anesthesiology community, the preoperative evaluation of patients by non-specialists appears to be a major desertion of our responsibilities as physicians. In April 2001, the Collège des médecins du Québec defined the core responsibilities of physicians as the diagnosis and treatment by pharmacological, surgical or other physical or psychological interventions. ${ }^{22}$ Confining our role to the delivery of anesthesia without having assessed patients ourselves (diagnosis) and prescribed/discussed the most appropriate anesthetic (treatment) will limit our specialty to merely its technical component.

Two recent Canadian articles seek to expand the role of non-anesthesia providers beyond their present limited activities given the ongoing concern of inadequate numbers of anesthesia personnel to provide the necessary services. A report by the Regional Perinatal Coordinating Committee in South Western Ontario has recommended investigating the potential of NA to provide analgesia during labour. ${ }^{23}$ The second, an article appearing in Canadian Nurse, has suggested a valuable role for NA in filling the void and meeting the required service needs. ${ }^{24}$ For the more "simple" cases, the delegation of anesthesia to a supervised assistant may well be appropriate. The presence of an anesthesiologist in the operating room remains essential for the more difficult/complex cases. As mentioned previously, this has worked well in several countries.

While there are many anesthesiologists who have strong feelings regarding the use of alternate providers we believe we need to face the realities and ensure that we play both an early and a leadership role in the provision of anesthesia services by non-physician providers, including the setting of standards and training of these individuals. Our input and collaboration with the authorities and other interested organizations ensures some measure of control over the quality of delivered anesthesia care.

In summary we have a well documented shortage of anesthesiologists. We need to work actively to achieve practical solutions to this shortage. The time has come for us to change focus if we want to achieve our objectives successfully.

\section{Les ressources médi- cales en anesthésie - il est temps d'ajuster l'objectif}

Au cours des cinq dernières années, cinq articles différents sur l'effectif médical en anesthésie sont parus dans le Journal canadien d'anesthésie (JCA). ${ }^{1-5}$ Chacun présentait un point de vue différent. Le premier, par Donen et coll., examinait le nombre total d'anesthésiologistes en exercice et reliaient les résultats aux indices médecin/population. ${ }^{1}$ Yang et coll. ont utilisé deux modèles pour évaluer les ressources médicales en anesthésie de l'Ontario, l'un fondé sur la demande, l'autre sur un seuil de référence. ${ }^{2}$ Byrick a abordé le problème en utilisant un modèle de dépenses «par habitant» comme mesure des services cliniques dispensés. ${ }^{3}$ Dans le même numéro, Craig et coll. ont combiné le modèle par habitant aux données de l'Association médicale canadienne et au nombre de 
diplômés du Collège royal des médecins et chirurgiens du Canada. ${ }^{4}$ Dans le présent numéro, Engen et ses collègues adoptent une toute autre méthode. Leur évaluation fondée sur la demande des besoins perçus en anesthésiologistes est le résultat d'une enquête hautement significative, avec un taux de réponse de $93 \%$, menée auprès de toutes les institutions de soins de santé du Canada qui offrent des services en anesthésie. ${ }^{5}$ Malgré ces différentes approches, le message est toujours le même. Il n'y a pas suffisamment d'anesthésiologistes pour répondre aux demandes actuelles et futures.

Ce problème n'est pas unique au Canada. Des rapports d'autres pays attestent de la même préoccupation. ${ }^{6-9} \mathrm{La}$ situation s'étend également à d'autres spécialités chirurgicales au Canada, confrontées à des pénuries similaires. ${ }^{\mathrm{A}} \mathrm{S}$ 'ajoutent à cela le nombre décroissant d'infirmières, au Canada et aux ÉtatsUnis, personnel qui fait intégralement partie des services d'anesthésie et de chirurgie. ${ }^{10,11}$ Un rapport récent de la British Columbia Medical Association a souligné la pénurie spécifique d'infirmières dans les salles d'opération, dans les salles de réveil et les unités de soins intensifs. ${ }^{12}$

D'autres facteurs ajoutent à la complexité et à la composition d'une future planification des ressources médicales. Etzioni, en regard de la population vieillissante aux États-Unis, a fait l'estimation conservatrice d'une hausse de $13 \%$ de la charge de travail actuelle en 2010 et de $30 \%$ en $2020 .^{13}$ En mai 2003, l'Association médicale mondiale a publié une déclaration sur le guide d'éthique pour le recrutement international de médecins en indiquant que les pays riches ne devraient pas drainer les ressources médicales des pays pauvres et du tiers monde pour recruter activement des médecins dans le but de combler leurs propres besoins. ${ }^{14}$ Nombre de ces pays pauvres ont commencé à exercer des pressions sur les gouvernements des pays riches, comme le Canada, pour qu'ils restreignent l'entrée de médecins étrangers. Un facteur supplémentaire, et peu documenté, est l'augmentation de la sévérité et de la complexité des cas d'anesthésie depuis 20 ans. Cette perception, partagée par quelques collègues, couplée à la charge de travail accrue le soir et la nuit, la redistribution des services chirurgicaux et anesthésiques par les autorités de la santé et une approche beaucoup plus impersonnelle du corps médical par les administrateurs ajoutent au

A Adams O. Canadian Specialty Society recommendations: Categorical summation. Canadian Medical Association. February 2, 2004. stress et à l'épuisement professionnel vécus aujourd'hui par de nombreux anesthésiologistes canadiens.

Étant donné la pénurie mondiale d'anesthésiologistes, nous croyons qu'il est temps de passer de la documentation des pénuries à la recherche de solutions qui vont maximiser la prestation et la distribution des services d'anesthésie. Le récent Sommet fédéral/provincial sur la santé à Ottawa, en septembre 2004, nous donne l'occasion d'agir dans ce sens. ${ }^{15,16}$ Nous l'envisageons comme une série de défis à relever par le milieu de l'anesthésie canadienne et des organismes comme la Société canadienne des anesthésiologistes (SCA), l'Association des anesthésiologistes du Québec et l'Association des services d'anesthésie des universités canadiennes.

\section{Défi 1}

Les organismes canadiens d'anesthésie devraient s'engager dans un processus national pour développer une approche normalisée de l'identification et de la collecte des données sur les ressources médicales en anesthésie aux fins de documentation des besoins au Canada

Rechercher des solutions efficaces ne veut pas dire abandonner la documentation des besoins. Toutes les approches utilisées jusqu'à maintenant ont contribué à nos connaissances. Cependant, nous devons maintenant développer un modèle de mesure uniforme. Cette mesure peut inclure quelques approches ou toutes les approches déjà utilisées. Nous devons nous entendre sur ce qui doit être mesuré et documenté, sur la facilité et le coût des mesures, la fiabilité et la fréquence des mesures. Il faut inclure les principaux acteurs comme le gouvernement et les planificateurs des services de santé. Il est évident que cette approche doit être spécifique à l'anesthésie.

\section{Défi 2}

Les organismes canadiens d'anesthésie, en plus de faire des pressions pour améliorer les conditions de travail des anesthésiologistes, doivent développer un programme de soins de santé complets pour les anesthésiologistes

Nous avons déjà fait allusion aux questions de stress et d'épuisement professionnel parmi nos collègues. Le personnel insatisfait n'est pas productif et ne demeure pas en poste. Il est aussi possible que la charge de travail accrue et le stress agissent négativement, sur la sécurité des patients, entre autres. En plus de vouloir améliorer l'environnement de travail, il faut développer des programmes de formation et de soutien pour aider l'anesthésiologiste à faire face au stress du quotidien. Ce qui comprend des techniques de résolution de conflits dans la salle d'opération et de gestion personnelle pour un meilleur mode de vie. Ces pro- 
grammes doivent être largement diffusés et appuyés.

\section{Défi 3}

Les services canadiens d'anesthésie doivent entreprendre et publier des études qui établissent un rapport entre l'efficacité des processus préopératoire, peropératoire et postopératoire.

Les cinq articles publiés dans le JCA réclament une augmentation des places de résidence en anesthésie. Malgré le fait qu'un certain nombre d'écoles de médecine augmentent leur contingent d'étudiants en médecine et en spécialité, on nous attribuera seulement, de façon réaliste, un petit nombre de places en résidence. Ce ne sera pas suffisant pour répondre à la demande attendue et croissante de services d'anesthésie. Nous devrons alors envisager d'autres options et façons de procéder.

Certains gains, petits mais significatifs, dans le nombre de cas traités par les anesthésiologistes peuvent être obtenus en définissant l'efficacité des services rendus en période préopératoire et périopératoire. De plus en plus d'articles sur la recherche en santé à l'étranger sont publiés. ${ }^{17,18} \mathrm{Il}$ y a sans doute un travail similaire entrepris par diverses institutions canadiennes. Cependant, il n'existe pas de documentation sur l'efficacité, ou l'inefficacité, des différentes stratégies tentées. L'Institut pour l'amélioration des soins de santé a été un important élément moteur dans le domaine de l'amélioration de la qualité. Des projets dirigés par des groupes de travail provenant de nombreuses institutions d'Amérique du Nord ont obtenu des résultats louables. ${ }^{19}$ Il est crucial pour nous d'étudier nos processus d'anesthésie et de chirurgie autant que de documenter et de partager les succès et les échecs. Pour réussir ces études, il faut les réaliser conjointement avec nos collègues de chirurgie et des soins infirmiers.

Au Québec, des assistants en anesthésie spécialement formés (AA ; membres de l'Ordre professionnel des inhalothérapeutes du Québec) jouent un rôle de soutien important depuis de nombreuses années. Qu'on pense à la préparation du matériel et à l'assistance de l'anesthésiologiste pendant l'induction de l'anesthésie et le réveil. Dans des situations choisies, ils peuvent superviser le cas seul après l'induction de l'anesthésie par l'anesthésiologiste qui doit, par ailleurs, demeurer immédiatement disponible. À noter qu'en 2004 la SCA a approuvé le rôle et le cadre des fonctions des AA et modifié leur Guide d'exercice de l'anesthésie pour tenir compte de ce fait. ${ }^{20}$ Récemment en Ontario, les AA ont commencé à offrir leur assistance en salle d'opération. Les partisans des AA décrivent leur valeur par de meilleurs services ren- dus. Etant donné la nécessité de justifier ce mode d'action, il pourrait être utile de faire une analyse de rentabilité de l'emploi des AA en salle d'opération.

Un domaine plus controversé à réexaminer est celui des indications pour la chirurgie et l'anesthésie. Le personnel disponible pour les services d'anesthésie étant limité, nous devons vérifier attentivement la valeur et l'efficacité de différentes interventions chez certains patients. C'est un domaine de pratique qui est étroitement aligné sur des normes de soins et l'amélioration de la qualité. Nous devons aborder et évaluer cet enjeu.

\section{Défi 4}

Les organismes canadiens d'anesthésie devraient adopter un rôle d'avant-garde dans l'exploration des possibilités et des actions du personnel non médical dans la prestation des services d'anesthésie.

Il y a, finalement, la question des autres prestateurs de soins dans les domaines de la technique et du monitorage. Avec le temps, les médecins se sont dégagés de ces responsabilités en les confiant à d'autres travailleurs de la santé et en leur donnant une responsabilité accrue d'intervention quand c'était approprié. Les exemples bien connus sont les techniciens médicaux de soins d'urgence pré-hospitaliers et le personnel infirmier des soins intensifs. Aux États-Unis, les «nurse anesthetists» (NA) fournissent des services en anesthésie depuis presque 120 ans. Aux États-Unis et dans de nombreux pays européens, leur fonction est soit indépendante, soit dépendante d'un anesthésiologiste. Ils font partie d'une équipe qui fournit des services d'anesthésie. L'application de la directive du temps de travail européen, qui limite le nombre d'heures de travail par semaine, a eu un effet significatif sur la capacité des anesthésiologistes à offrir des services de qualité. En conséquence, un certain nombre de pays pensent sérieusement à introduire les NA pour résoudre le problème des ressources médicales. Actuellement, au Royaume-Uni, certains projets pilotes utilisant les NA sont en cours. ${ }^{7,21}$ Les organismes d'anesthésiologie travaillent étroitement avec les autorités pour s'assurer que les programmes de formation, les normes de soins et la prestation future de soins anesthésiques soient fortement liés à leurs propres organismes. À cet effet, ils cherchent à éviter l'erreur faite par l'American Society of Anesthesiologists au début du siècle dernier alors qu'il ont tenté de miner les efforts des NA. Cette attitude a abouti à l'établissement ultérieur d'une organisation et d'un mécanisme d'accréditation indépendants pour les NA.

Au Canada, il y a aussi un léger mouvement dans ce sens. Les consultations pré-admission emploient de plus en plus de personnel spécialement formé pour 
faire des évaluations préopératoires. De nombreux hôpitaux sans vocation pédagogique comptent maintenant sur les médecins de famille pour recueillir les renseignements préopératoires et faire l'examen physique. Le plus souvent, l'anesthésiologiste rencontre le patient pour la première fois quelques minutes littéralement avant l'anesthésie. Même si elle est bien acceptée par les anesthésiologistes, l'évaluation préopératoire faite par des non spécialistes apparait comme un abandon de nos responsabilités de médecins. En avril 2001, le Collège des médecins du Québec a défini les principales responsabilités des médecins comme étant le diagnostic et le traitement par des interventions pharmacologiques, chirurgicales ou autres actions physiques ou psychologiques. ${ }^{22}$ Confiner notre rôle dans l'administration de l'anesthésie sans avoir évalué le patient nous-mêmes (diagnostic) et avoir prescrit l'anesthésique le plus approprié ou en avoir discuté (traitement) va limiter notre spécialité à sa simple composante technique.

Dans deux récents articles canadiens, on a tenté d'élargir le rôle des non spécialistes en anesthésie au delà des limites de leurs activités présentes, étant donné le souci constant du nombre insuffisant de personnel d'anesthésie pour rendre les services nécessaires. Un rapport du Regional Perinatal Coordinating Committee in South Western Ontario recommandait d'évaluer la possibilité que les NA puissent assurer l'analgésie pendant le travail. ${ }^{23} \mathrm{Un}$ second article, paru dans Canadian Nurse, était en faveur d'un rôle appréciable pour les NA qui pourraient combler le vide et répondre aux demandes de service nécessaires. ${ }^{24}$ Pour les cas les plus «simples», la délégation de l'anesthésie à un assistant supervisé pourrait être pertinente. La présence d'un anesthésiologiste en salle d'opération demeure essentielle pour les cas plus difficiles ou complexes. Comme mentionné précédemment, cet arrangement fonctionne bien dans certains pays.

Il y a un fort courant d'opinion parmi les anesthésiologistes au sujet des non spécialistes en anesthésie, mais nous croyons que nous devons faire face à la réalité et nous assurer d'intervenir tôt et d'adopter un rôle d'avant-garde dans la prestation des services d'anesthésie par des non-médecins. Ce qui comprend l'élaboration de normes et la formation de ces intervenants. Nos suggestions et notre collaboration avec les autorités et autres organismes intéressés peuvent assurer un certain contrôle sur la qualité des services rendus en anesthésie.

En résumé, nous avons une pénurie d'anesthésiologistes bien documentée. Nous devons travailler activement pour trouver des solutions pratiques à ce pro- blème. Le temps est venu d'ajuster l'objectif si nous voulons réussir à atteindre nos buts.

\section{References}

1 Donen N, King F, Reid D, Blackstock D. Canadian anesthesia physician resources: 1996 and beyond. Can J Anesth 1999; 46: 962-9.

2 Yang H, Byrick R, Donen N. Analysis of anesthesia physician resources: projected Ontario deficit in 2005. Can J Anesth 2000; 47: 179-84.

3 Byrick RJ, Craig D, Carli F. A physician workforce planning model applied to Canadian anesthesiology: assessment of need. Can J Anesth 2002; 49: 663-70.

4 Craig D, Byrick R, Carli F. A physician workforce planning model applied to Canadian anesthesiology: planning the future supply of anesthesiologists. Can J Anesth 2002; 49: 671-7.

5 Engen DA, Morewood GH, Ghazar NJ, Ashbury T, VanDenKerkhof EG, Wang L. A demand-based assessment of the Canadian anesthesia workforce - 2002 through 2007. Can J Anesth 2005; 52: 18-25.

6 Schubert A, Eckhout $G$ Jr, Tremper K. An updated view of the national anesthesia personnel shortfall. Anesth Analg 2003; 96: 207-14.

7 The role of non-medical staff in the delivery of anaesthesia services. Position paper. The Royal College of Anaesthetists, October 2002.

8 Pontone S, Brouard N, Scherpereel P, Boulardl G, Arduin P; CFAR-SFAR-INED Working Group. Demography of French anaesthesiologists. Results of a national survey by the French College of Anaesthesiologists (CFAR) and the French National Society of Anaesthesia and Intensive Care (SFAR), supported by the National Institute for Demographic Studies (INED). Eur J Anaesthesiol 2004; 21: 398-407.

9 Demeere JL. Anaesthesia manpower in Belgium until 2020: nurse anaesthetists- a possible solution? Eur J Anaesthesiol 2002; 19: 755-9.

10 Health Personnel trends in Canada 1993-2002. Canadian Institute for Health Information, April 2004.

11 Bednash $G$. The decreasing supply of registered nurses: inevitable future or call to action? JAMA 2000; 283: 2985-7.

12 Specialty care in BC: a system in distress. A discussion paper by BC's physicians. British Columbia Medical Association, June 2004. Available from URL; http://www.bcma.org (accessed June 10, 2004).

13 Etzioni DA, Liu JH, Maggard MA, Ko CY. The aging population and its impact on the surgery workforce. Ann Surg 2003; 238: 170-7.

14 The World Medical Association statement on ethical guidelines for international recruitment of physicians. World Medical Association, Helsinki, May 2003. 
15 \$41.2-billion health deal struck. Times Colonist, Victoria, September 16, 2004.

1618 millards pour la santé. Cyberpresse, 16 septembre 2004; www.cyberpresse.ca

17 Epstein RH, Dexter F. Statistical power analysis to estimate how many months of data are required to identify operating room staffing solutions to reduce labor costs and increase productivity. Anesth Analg 2002; 94: 640-3.

18 Dzoljic M, Zimmerman M, Legemate D, Klazinga NS. Reduced nurse working time and surgical productivity and economics. Anesth Analg 2003; 97: 1127-32.

19 Smoothing elective surgical admissions. Institute for Health Care Improvement. Available from URL; http://www.qualityhealthcare.org/IHI/Topics/Flow/ PatientFlow/EmergingContent/SmoothingElectiveSur gicalAdmissions.htm (accessed June 25, 2004).

20 Anesthesia assistants. Implications of the recent changes to the CAS Guidelines. Anesthesia News. Canadian Anesthesiologists' Society, May 2004.

21 Sbaih L. New ways of working in anaesthesia.

Changing workforce programme, NHS modernization agency. Available from URL;

http://www.modern.nhs.uk/workingtime/nwwanaes. pdf Accessed June 25, 2004.

22 Collège des médecins du Québec. L'exercice de la médecine et les rôles du médecin au sein du système professionnel: énoncé de position du Collège des médecins du Québec, Avril 2001.

23 At risk: perinatal services in Southwestern Ontario. Issue summary and recommendations. South Western Regional Perinatal Services project, December 2003.

24 Schreiber R, MacDonald M. Nurse anesthesia. The time has come. Can Nurse 2003; 99: 20-3. 